\title{
Effect of Different Methods for the Overhang Amalgam Restorations Removal on Periodontal Health Status
}

\author{
Dana M. Yawer Aldalawi ${ }^{1 *}$, Dara H. Saeed ${ }^{2}$, Sangar H. Ali ${ }^{3}$ \\ ${ }^{1}$ Department of Periodontic, Khanzad Teaching Center, Erbil Directorate of Health, Ministry of Health, Erbil, Kurdistan Region, Iraq, \\ ${ }^{2}$ Department of Conservative, Hawler Medical University, Erbil, Kurdistan Region, Iraq, ${ }^{3}$ Department of Oral and Maxillofacial Radiology, \\ Khanzad Teaching Center, Erbil Directorate of Health, Ministry of Health, Erbil, Kurdistan Region, Iraq
}

\author{
*Corresponding author: \\ Dana M. Yawer Aldalawi, \\ Department of Periodontic, \\ Khanzad Teaching Center, \\ Erbil Directorate of Health, \\ Ministry of Health, Erbil, \\ Kurdistan Region, Iraq. \\ E-mail: danadalawy@yahoo. \\ com
}

Received: 16 November 2019

Accepted: 20 January 2020

Published: 30 June 2020

DOI

10.25156/ptj.v10n1y2020.pp61-67

\section{A B S T R A C T}

Overhanging tooth restorations due to environmental change of the balance between beneficial bacteria and periodontopathogens lead to periodontal breakdown. This study aim was to evaluate the periodontal status after using different techniques for amalgam overhang removal. The proximal restorations were examined clinically and then radiographically by taking bitewing radiograph. From a total of 1200 patients examined in College of Dentistry, Hawler Medical University and (Khanzad) Specialized Centre for Dentistry for presence of proximal amalgam restoration, for this interventional and prospective study, 45 patients with amalgam overhang were divided into three groups each 15 patients according to size and position of amalgam overhang each had one type of the following treatment (Refilling, Diamond Bur, EVA system) and 15 patients without amalgam overhang (control group) selected completed the trial. Clinical periodontal parameters were measured in teeth with amalgam overhang at baseline, 2 week, 1 and 3 months after removal of amalgam overhang. The results showed that the periodontal parameters showed a gradual decrease in all groups throughout the follow-up visits (except calculus index) and became a statistically significant in the 3 months follow-up following treatment $(P<0.001)$, with more reduction found in refilling group followed by EVA system then diamond bur group. In conclusion that it is better to replace the restoration rather than to use the other methods of treatment according to periodontal health which recorded by periodontal parameters.

Keywords: Amalgam overhang; Periodontal parameters; Bitewing X-ray; Plaque; Calculus

\section{INTRODUCTION}

Overhanging tooth restorations can become a risk factor for periodontal degredation due to the formation of environmental disturbance of the balance between beneficial bacteria and periodontopathogens (Nunn, 2003).

Studies have shown that in overhang teeth restorations there is more periodontal breakdown and inflammation than those without. Overhangs cause an increase in plaque formation (Kells and Linden, 1992; Lervik et al., 1984), and a shift in the microbial composition (Lang et al., 1983) from normal flora to one characteristic of periodontal disease.

Several factors can affect the periodontal disease course. Along with systemic factors, it is widely accepted that local factors such as overhanging restorations may lead to loss of the alveolar bone crest (Al-Farhan, and Al-Shammari, 2008).

There is a good documentation that a gingival inflammation, pocket depth, attachment loss, and loss of bone increased in areas adjacent to overhang dental restorations (ODR) (Claman et al., 1986; Chen et al. 1987).
Evidence exists that any subgingival overhang restorations are found that there are some degree of inflammation in both histological and clinical examinations (Karlsen, 1970; Trivedi and Talim, 1973), and associated with high gingival fluid flow (Mörmann et al., 1974; Mannerberg, 1971).

The effect of amalgam overhang restoration is to worsen these responses by increasing the plaque retention likely. In animal model systems show that plaque accumulation leads to the development of gingival inflammation into the periodontal tissues (Lindhe et al., 1973).

Most studies report a high level of gingival inflammation and adequate alveolar bone resorption associated with the amalgam overhangs (Rodriguez-Ferrer et al., 1980; Gilmore et al., 1971).

Removal of overhang amalgam restoration stimulates the hygienic phase effect of the periodontal therapy and results in reduction of the gingival inflammation and periodontal destruction (Rodriguez-Ferrer et al., 1980).

Searching data base of researches countersink, no previous studies have been done or published yet in Kurdistan 
region about the effect of amalgam overhang removal on the health of periodontal tissue.

Hence, the aim of the present study was to determine the short-term effect of overhang removal of posterior amalgam restoration by different methods on the periodontal health status through evaluating the following clinical periodontal parameters (plaque index [PLI], gingival index [GI], calculus index [CALI, bleeding on probing [BOP], and probing pocket depth [PPD]).

\section{MATERIALS AND METHODS}

Sixty patients were involved in the study from a total of 1200 patients (among 18-55 years old) examined in both (College of Dentistry) Hawler Medical University and (Khanzad) Specialized Centre for Dentistry examined clinically for the presence of proximal restorations; divided into four groups, on the basis of the presence of amalgam overhang or not; amalgam overhang size and the amount of the bone resorption (PPD not $<3 \mathrm{~mm}$ ).

The ethical approvals for conducting the study and sample selection were obtained from the Ethical Committee of research in College of Dentistry/Hawler Medical University. Informed consent to participate and maintain confidentiality was observed. Before data collection researcher explained the objectives of this study and request consent for participation in the study.

Inclusion criteria regarding the tooth selection included:

All proximal premolars and molars (excluding third molar) with Class II amalgam restorations were included and examined clinically (using dental mirror and explorer) and radiographically (using bite-wing radiograph) to detect amalgam restorations which have overhanging margin.

Exclusion criteria regarding the tooth selection included:

1. Amalgam overhangs for $<6$ months were not included in the study

2. At the time of overhang correction, surfaces with an overhang should have a periodontal pocket depth PPD between 3 and $6 \mathrm{~mm}$ using William's periodontal probe by inserting it the direction parallel to the long axis of the tooth within the sulcus when no pressure applied. The probe should be allowed to fall by its own weight then the measurements are taken to the nearest millimeters according to Claman et al. (1986) and Jansson et al. (1994).

The approximal restoration was considered as possessing an overhang when:
1. Exact interruption in the coronal movement of the dental probe at the tooth-amalgam contact according to Arneberg et al. (1980), and /or

2. A separate ledge of radiopaque amalgam restoration which did not conform to the natural contours of the teeth, only visible and distinct ledges in bite-wing radiographs were included as overhangs as described by Gorzo et al. (1979) and Arneberg et al. (1980).

Out of 1200 Kurdish patients, 60 subjects with amalgam overhang (33 males and 27 females) were chosen according to the criteria of the sample selection mentioned above with different groups of treatment divided into four groups each group involve 15 patients according to the type of amalgam overhang treatment (EVA, diamond bur, refilling, and control).

All participants were carefully informed about the aim of the investigation and procedure which they were free to alienate at any time during the study.

The oral examination was conducted to evaluate the periodontal status and the restorative quality. Sites with ODRs were recorded.

Selection of the patients according to that they did not receive oral hygiene instructions before at least 6 months, so at the first appointment the baseline examination, each was instructed in proper oral hygiene, motivation including education on the nature of periodontal disease and the effect of the defective restoration on the incidence and severity.

All patients received supra-gingival prophylaxis also calculus in close contact with ODR was removed (Rodriguez-Ferrer et al., 1980).

At the baseline appointment, the periodontal parameters were recorded and then the overhanging margins were managed by one of the following procedures:

1. Replacement of the whole restoration with new amalgam restoration was had been done when there were a large amalgam overhanging and difficult to be removed by other methods

2. EVA Directory System is planned to move up and down as well as all around for maximum access and workability and the diamond and plastic tips were provided in a variety of sizes and thickness to adapt to interproximal spaces [Figure 1]

3. Removal by conventional finishing diamond burs (Hi-Di ISO \# 197/014/England) in an air-motor slow handpiece; they are found in a variety of shapes and sizes pattern. It is recommended that a four or six finishing burs should be used in the burnishing direction rather than cutting direction. 


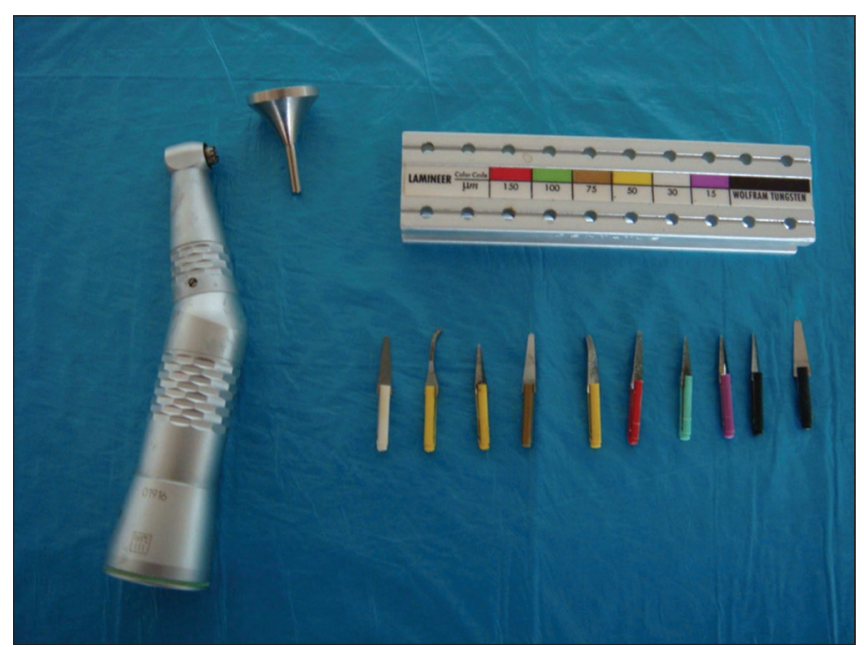

Figure 1: EVA instrument system (Profin, W\&H)

Before baseline clinical examinations, the aims of the study were clearly declared and the process of the research was announced so that every patient knew exactly what it is all about to assure full cooperation (and that it would include clinical and radiographic examination of the tooth/teeth included in the study).

The baseline clinical examinations were performed in the periodontology dental clinic for each patient under the study by the researcher and one interexaminer to ensure interexaminer reliability and he was blinded to the groups.

Plaque, calculus, gingival condition, $\mathrm{BOP}$, and $\mathrm{PPD}$ were recorded using a manual periodontal probe (Williams probe) at 4 areas per tooth (mesial, distal, buccal, and lingual), then using mean of each tooth for statistical analysis. Interproximal probing is done from the buccal area of the tooth as close as possible to the contact point with the probe tip in a direction parallel to the long axis of the tooth; measurements were made to the nearest millimeters by selecting the site of amalgam overhang.

The bite-wing radiograph represents that the premolar and molar areas of both right and left side were done. The digital bite-wing radiograph (Planmeca Pro One) was operated at $68 \mathrm{kVp}, 7 \mathrm{~mA}, 9.5 \mathrm{~s}$ exposure time according to the individual size [Figure 2].

Before starting the study and for the goal of calibration, training and testing of the design; a pilot study was used about 2-3 weeks before the direction of actual project on nine patients with teeth restored with amalgam (overhang). Divided into three groups (each group three patients) first group overhang amalgam refilled with amalgam and second group ODRs removed by EVA reciprocating motor driven system and last one ODRs removed by diamond tapered

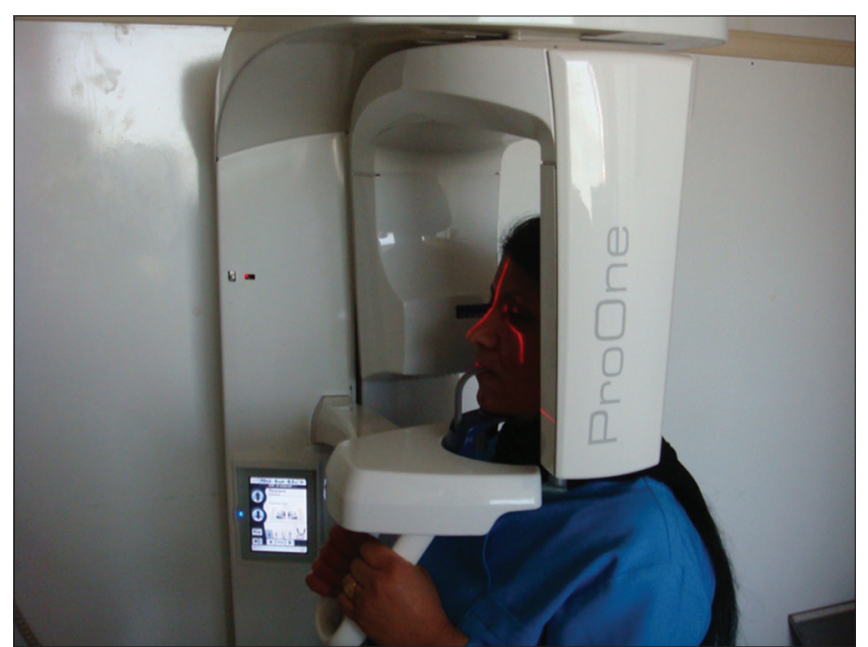

Figure 2: Digitalized panoramic radiograph machine taking bitewing radiograph

finishing bur, and all groups examined both clinically and radiographically [Figures 3-8].

The interexamination calibration was performed by the researcher and an expert periodontal specialist for all clinical parameters. The calibration was made for interproximal surfaces of posterior teeth except third molar and distal surface of second molars. Paired t-test results showed no significant differences between the two readings for interexaminer reliability statically at $P>0.05$. 1. Comparison was done between baseline reading (of the studied indices) with the readings after 14 days, 30 days, and 90 days using students' $t$-test. Paired $t$-test was applied to test the significant differences between the mean of the following:

- Intraexaminer calibration

- Interexaminer calibration.

Periodontal indices in baseline visit and each following visits.

2. The comparison between the mean of the measured differences was done between the four studied groups using analysis of ANOVA. Variance (ANOVA) test one-way analysis was used to detect statistically significant dissimilarity between different groups

3. After detecting a significant difference by ANOVA test, test least significant difference (LSD) was applied to determine which means were different from other.

\section{RESULTS AND DISCUSSION}

The differences in periodontal parameters between the different groups are shown in Table 1 using one-way analysis of mean variance (ANOVA). LSD test was applied to examine, the significant differences between each two groups are shown in Table 2. 
Table 1: ANOVA table for the periodontal parameters of all groups

\begin{tabular}{|c|c|c|c|c|c|c|c|c|c|}
\hline \multirow[t]{2}{*}{ Variable (month) } & \multicolumn{2}{|c|}{ Control } & \multicolumn{2}{|c|}{ Bur } & \multicolumn{2}{|c|}{ Refilling } & \multicolumn{2}{|c|}{ EVA system } & \multirow[t]{2}{*}{$P$-value } \\
\hline & Mean & S.D. \pm & Mean & S.D. \pm & Mean & S.D. \pm & Mean & S.D. \pm & \\
\hline PLI 3 & 1.23 & 0.26 & 0.97 & 0.13 & 0.96 & 0.09 & 1.07 & 0.18 & $0.025^{\star}$ \\
\hline GI 3 & 1.02 & 0.15 & 1.37 & 0.16 & 0.95 & 0.14 & 1.12 & 0.16 & $0.05^{*}$ \\
\hline BOP 3 & 0.78 & 0.23 & 0.97 & 0.09 & 0.71 & 0.09 & 0.87 & 0.13 & $0.001^{*}$ \\
\hline PPD 3 & 1.87 & 0.99 & 3.53 & 0.74 & 3.57 & 0.64 & 3.53 & 0.63 & $0.041^{*}$ \\
\hline
\end{tabular}

*Significant at $P \leq 0.05$

Table 2: LSD after ANOVA table of the periodontal parameters for groups

\begin{tabular}{|c|c|c|c|c|c|c|c|c|c|c|c|c|}
\hline \multirow[t]{2}{*}{ Variable } & \multicolumn{2}{|c|}{ Control and bur } & \multicolumn{2}{|c|}{$\begin{array}{l}\text { Control and } \\
\text { refilling }\end{array}$} & \multicolumn{2}{|c|}{$\begin{array}{c}\text { Control and EVA } \\
\text { system }\end{array}$} & \multicolumn{2}{|c|}{$\begin{array}{l}\text { Bur and } \\
\text { refilling }\end{array}$} & \multicolumn{2}{|c|}{$\begin{array}{l}\text { Bur and EVA } \\
\text { system }\end{array}$} & \multicolumn{2}{|c|}{$\begin{array}{c}\text { Refilling and EVA } \\
\text { system }\end{array}$} \\
\hline & $P$-value & LSD & $P$-value & LSD & $P$-value & LSD & $P$-value & LSD & $P$-value & LSD & $P$-value & LSD \\
\hline PLI 3 months & $0.00^{* *}$ & 0.26 & $0.00^{* *}$ & 0.27 & $0.01^{*}$ & 0.16 & 0.97 & 0 & 0.12 & -0.1 & 0.12 & 0.1 \\
\hline GI 3 months & $0.00^{* *}$ & 0.35 & $0.00^{* *}$ & 0.78 & 0.08 & 0.1 & $0.00^{* *}$ & 0.42 & $0.00^{* *}$ & 0.25 & $0.04^{*}$ & 0.17 \\
\hline $\begin{array}{l}\text { BOP } 3 \\
\text { months }\end{array}$ & $0.01^{*}$ & 0.18 & 0.21 & 0.06 & 0.12 & 0.08 & $0.00^{* *}$ & 0.25 & 0.06 & 0.1 & $0.00^{* *}$ & 0.15 \\
\hline $\begin{array}{l}\text { PPD } 3 \\
\text { months }\end{array}$ & $0.00^{* *}$ & 1.67 & $0.00^{* *}$ & 1.7 & $0.00^{* *}$ & 1.67 & 0.83 & 0.03 & 0.1 & 0 & 0.89 & 0.03 \\
\hline
\end{tabular}

*Significant at $P \leq 0.05$

The findings of the present study cannot be compared with any other studies since there are no any available references or study about periodontal parameters in ODR removal for such groups. The PLI showed a significant difference which results from that was a significant reduction in PLI for bur, refilling, and EVA system when compared to control group which was due to that in ODR groups the underlining cause of increased PLI were removed and patient aware of their problem and how to restricted to the instructions while in control group there may be patients not restricted to oral hygiene instruction since the patients not having a problem that they complain from and the following researchers reported more access for plaque removal by the patients after overhang removal (Brunsvold and Lane, 1990; Gorzo et al., 1979; Highfield and Powell, 1978) since the removal of overhang amalgam leave a sufficiently smooth surface to allow efficient plaque removal by patient (Lim and Ong, 1989).

The PPD index in three month follow-up showed a significant difference $(P<0.05)$, which results from that was a significant decreasing in PPD index for control group when liken to refilling, Bur, and EVA system groups while there was no significant reduction among refilling, Bur, and EVA system groups; which all groups showed a significant reduction nearly similar effect on the periodontal pocket with amalgam overhang when compared to control group (Gorzo et al., 1979; Claman et al., 1986; Roman-Torres et al., 2006; Mokeem, 2007).

\section{PLI}

The PLI in 3 month follow-up showed a significant difference in ANOVA as shown in Table $1(P \leq 0.05)$ which resulted from a significant reduction in PLI for Bur, refilling, and EVA system when liken to control group, as shown in Table $2(P \leq 0.05)$. Although the patients were managed to maintain a high quality of plaque removal which was reinforced along the study period after overhang removal, most of the subjects were evidently capable of maintaining an excellent plaque control for a considerable period of time without any need of professional support, mean plaque PLI scores fluctuated between improvement and minor deterioration, still below 0.5 at all times in all groups.

This result agreed with Mokeem (2007); Renggli and Regolati (1972); and Leon (1976) they showed that PLI had been shown improvement in the subsequent visits after amalgam overhang removal combined with oral hygiene improvement because such amalgam overhangs and uneven proximal surfaces, devote to plaque accumulation and gingival inflammation, but Highfield and Powell (1978); Rodriguez-Ferrer et al.' Strahan and Newman (1980); and Roman-Torres et al. (2006) showed that removal of overhanging margins did not, however, result in a significant improvement in plaque scores for these surfaces, though a general improvement was noted in the predicted direction. This may be due to the low number of patients involved, or to the inadequacies of the plaque removal.

\section{GI}

The GI in 3 month follow-up showed a significant difference in ANOVA. as shown in Table $1(P \leq 0.05)$, which resulted from a significant reduction in GI for refilling group when compared to control, bur. and EVA system groups and also there is a significant increased for the bur group when compared to control and EVA system groups. The EVA system when compared to bur group showed decrease in GI, as shown in Table $2(P \leq 0.05)$. In the present study, baseline examination invariably showed that gingival inflammation in relation to amalgam 
overhang. Most of the studies reported significantly more inflammation accompanied with overhanging amalgam restorations which agreed with the present study (Gorzo et al., 1979; Lang et al., 1983; Eid, 1987). During the follow-up visits, it was noted that the GI was decreasing gradually in subsequent visits follow-up ( 2 weeks, 1 month, and 3 months) in all groups.

\section{CALI}

Throughout the study period, there was no calculus formation. From the starting of the study, a thorough scaling and polishing were performed for all patients participated in the study and until the end of the study period which was 3 months (short period); there was no calculus formation on the amalgam overhang restored teeth.

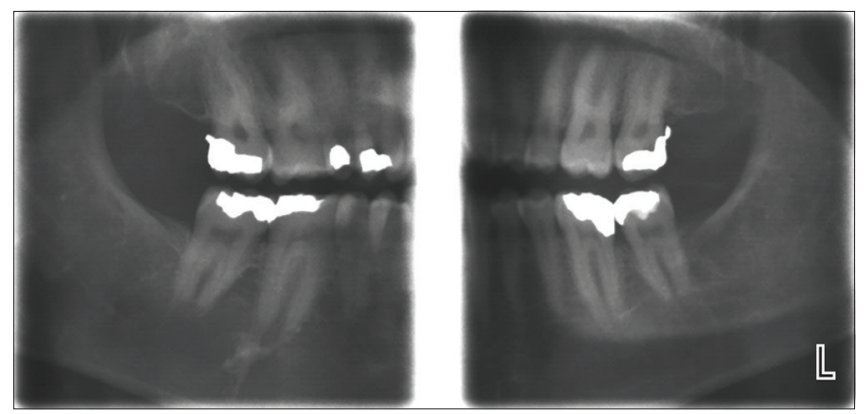

Figure 3: Bite-wing radiograph of amalgam overhang for both teeth (No.2 and 17)

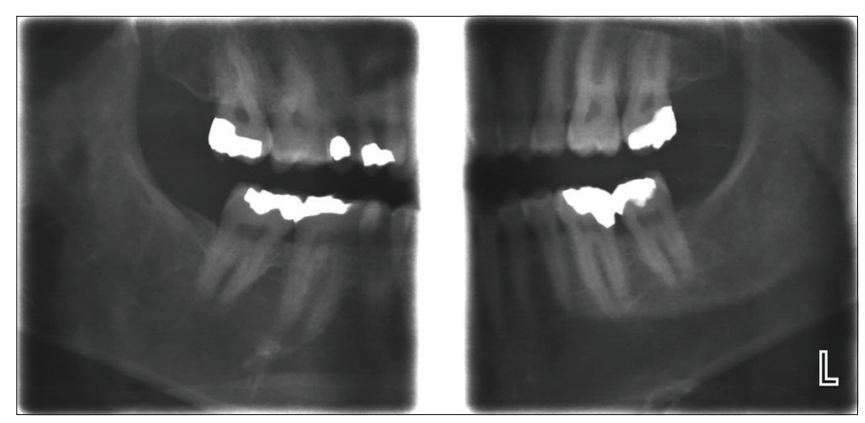

Figure 4: Bite-wing radiograph of amalgam overhang for both teeth (No.2 and 17) after removal by diamond finishing bur

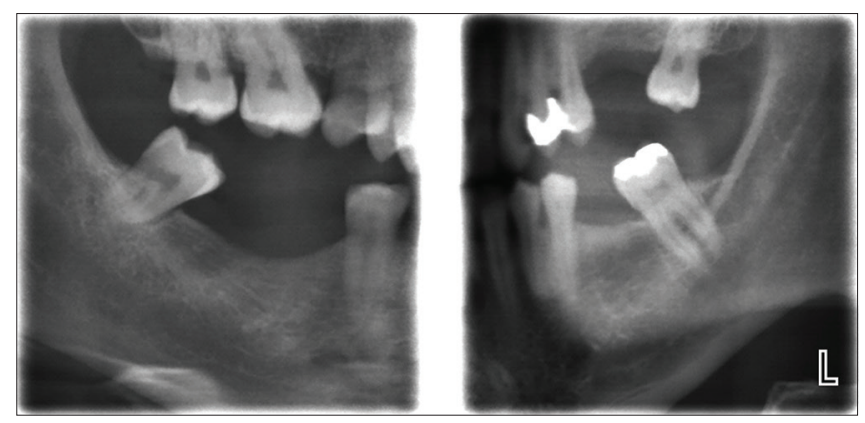

Figure 5: Bite-wing radiograph of amalgam overhang for tooth (No.12)

\section{BOP Index}

The BOP index in three month follow-up showed a significant difference in ANOVA, as shown in Table 1 $(P \leq 0.05)$, which resulted from a clearly reduction in BOP index for Bur when compared to control group and also there is a notable decrease for the refilling group when compared to bur and EVA system groups. The EVA system when compared to Bur group showed no significant decrease in BOP index, as shown in Table $2(P>0.05)$. The following studies that agreed with these findings (e.g., Mokeem, 2007; Highfield and Powell, 1978; Gullo and Powell, 1979; Gorzo et al., 1979; Renggli and Regolati, 1972 and Leon, 1976; Lightner et al., 1971; Suomi et al., 1971; Axelsson and Lindhe 1974) all of these studies showed a significant difference between overhang removed teeth and control teeth due to reduction in the gingival inflammation,

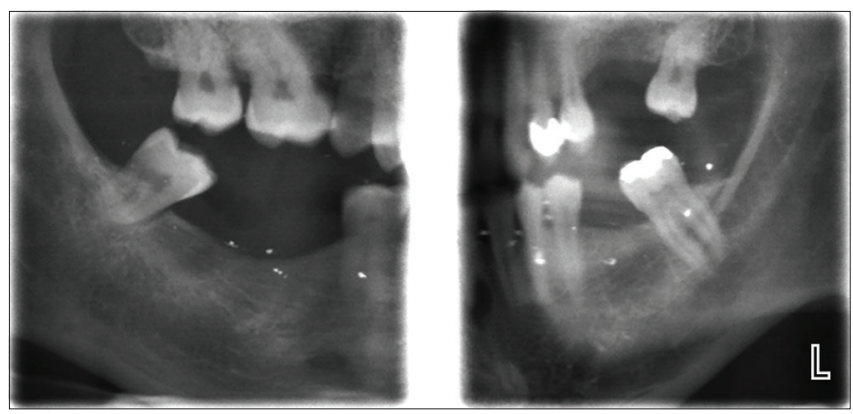

Figure 6: Bite-wing radiograph of amalgam overhang for tooth (No.12) after replacement by new amalgam restoration

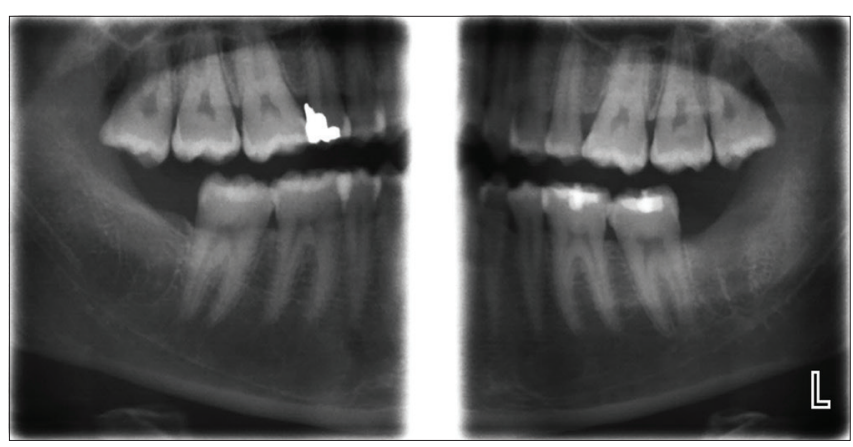

Figure 7: Bite-wing radiograph of amalgam overhang for tooth (No.4)

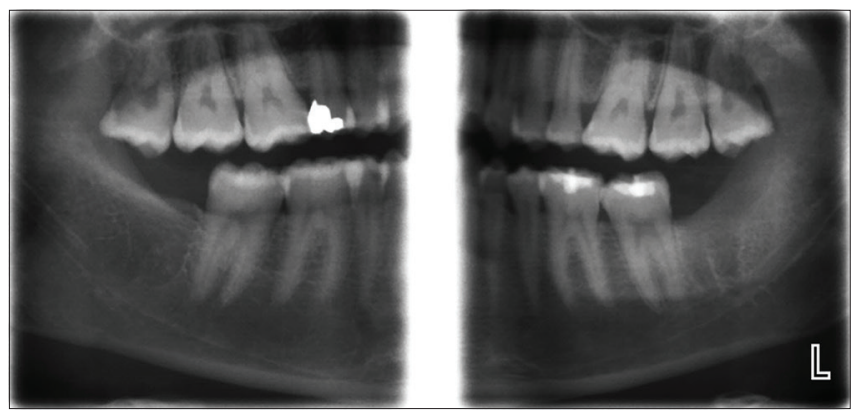

Figure 8: Bite-wing radiograph of amalgam overhang after removal for tooth (No.4) by EVA system 
followed by amalgam overhang removal. Roman-Torres et al. (2006) and Rodriguez-Ferrer et al. (1980) studies disagreed with the results. There may be due to different methodology, sample size, the criteria, or measurements which may be not standardized.

\section{PPD Index}

The PPD index in 3 months follow-up showed a significant difference in ANOVA, as shown in Table $1(P \leq 0.05)$, which resulted was a remarkable decease in PPD index for control group when equate to refilling, bur, and EVA system groups, while there is no significant reduction among refilling, bur, and EVA system groups, as shown in Table $2(P>0.05)$. The following studies which agreed with this were Mokeem (2007); Claman et al. (1986); Gorzo et al. (1979); Highfield and Powell (1978), Roman-Torres et al. (2006); while agreement with results of short-term studies conducted on the removal of overhangs but in one month visit appointment after active treatment (Gorzo et al., 1979; Rodriguez-Ferrer et al., 1980) which may be due to hygienic phase of periodontal therapy. While studies disagreed with this result were Turgeon et al. (1972) and Leon (1976) Keszthelyi and Szabo (1984).

\section{CONCLUSIONS}

1. The follow-up visits of different methods for amalgam overhang removal revealed a notable reduction in the plaque and gingival indices when compared to baseline visit

2. The follow-up visits for (refilling and EVA system) methods of removal of amalgam overhang showed a significant reduction in the BOP index when compared to baseline visit, while diamond bur method showed no significant reduction in the BOP in the subsequent visits

3. The findings reveal much more reduction in all periodontal parameter in 3 months

4. The PLI showed that was a significant reduction in PLI for bur, refilling, and EVA system when compared to control group and a significant reduction in GI for refilling group when compared to control, bur, and EVA system groups and also there is a significant increased for the Bur group when compared to control and EVA system groups

5. The PPD showed that was a significant reduction in probing pocket depth index for control group when compared to refilling, bur, and EVA system groups.

\section{REFERENCES}

Al Farhan, K. and K. F. Al-Shammari. 2008. The effect of amalgam overhangs on alveolar bone loss. Saudi Med. J. 21(2): 155-160.
Arneberg, P., J. Silness and H. Nordbø. 1980. Marginal fit and cervical extent of Class II amalgam restorations related to periodontal condition: A clinical and roentgenological study of overhang elimination. J. Periodontal Res. 15(6): 669-677.

Axelsson, P. and J. Lindhe. 1974. The effect of a preventive programme on dental plaque, gingivitis and caries in schoolchildren. Results after one and two years. J. Clin. Periodontol. 1(2): 126-138.

Brunsvold, M. A. and J. J. Lane. 1990. The prevalence of overhanging dental restorations and their relationship to periodontal disease. J. Clin. Periodontol. 17(2): 67-72.

Chen, J. T. J., J. G. Burch, F. M. Beck and J. E. Horton. 1987. Periodontal attachment loss associated with proximal tooth restorations. J. Prosthet. Dent. 57(4): 416-420.

Claman, L. J., P. T. Koidis and J. G. Burch. 1986. Proximal tooth surface quality and periodontal probing depth. J. Am. Dent. Assoc. 113(6): 890-893.

Eid, M. 1987. Relationship between overhanging amalgam restorations and periodontal disease. Quintessence Int. 18: 775-781.

Gilmore, N. and A. Sheiham. 1971. Overhanging dental restorations and periodontal disease. J. Periodontol. 42: 8-12.

Gorzo, I., H. N. Newman and J. D. Strahan. 1979. Amalgam restorations, plaque removal and periodontal health. J. Clin. Periodontol. 6(2): 98-105.

Gullo, C. A. O. and R. N. Powell. 1979. The effect of placement of cervical margins of Class II amalgam restorations on plaque accumulation and gingival health. J. Oral Rehabil. 6(4): 317-322.

Highfield, J. E. and R. N. Powell. 1978. Effects of removal of posterior overhanging metallic margins of restorations upon the periodontal tissues. J. Clin. Periodontol. 5(3): 169-181.

Jansson, L., H. Ehnevid, S. Lindskog and L. Blomlöf. 1994. Proximal restorations and periodontal status. J. Clin. Periodontol. 21(9): 577-582.

Karlsen, K. 1970. Gingival reactions to dental restorations. Acta Odontol. Scand. 28(6): 895-904.

Kells, B. E. and G. J. Linden. 1992. Overhanging amalgam restorations in young adults attending a periodontal department. J. Dent. 20(2): 85-89.

Keszthelyi, G. and I. Szabo. 1984. Influence of Class II amalgam fillings on attachment loss. J. Clin. Periodontol. 11(2): 81-86.

Lang, N. P., R. A. Kiel and K. Anderhalden. 1983. Clinical and microbiological effects of subgingival restorations with overhanging or clinically perfect margins. J. Clin. Periodontol. 10(6): 563-578.

Leon, A. R. 1976. Amalgam restorations and periodontal disease. Br. Dent. J. 140(11): 377-382.

Lervik, T., P. J. Riordan and O. Haugejorden. 1984. Periodontal disease and approximal overhangs on amalgam restorations in Norwegian 21-year-olds. Community Dent. Oral Epidemiol. 12(4): 264-268.

Lightner, L. M., T. J. O'Leary, R. B. Drake, P. P. Crump and M. F. Allen. 1971. Preventive periodontic treatment procedures: Results over 46 months. J. Periodontol. 42(9): 555-561.

Lim, K. C. and G. H. Ong. 1989. Methods of proximal amalgam overhang removal-a comparison of different techniques. Ann. Acad Med. Singapore. 18(5): 599-602.

Lindhe, J., S. E. Hamp, and H. Löe. 1973. Experimental periodontitis in the beagle dog. J. Periodontal Res. 8(1): 1-10.

Mannerberg, F. 1971. Gingival changes following porcelain crown therapy. Odontol. Revy. 22: 155-162.

Mokeem, S. A. 2007. The impacts of amalgam overhang removal on periodontal parameters and gingival crevicular fluid volume. 
Pak. Oral Dent. J. 27(1): 17-22.

Mörmann, W., B. Regolati and H. H. Renggli. 1974. Gingival reaction to well-fitted subgingival proximal gold inlays. J. Clin. Periodontol. 1(2): 120-125.

Nunn, M. E. 2003. Understanding the etiology of periodontitis: An overview of periodontal risk factors. Periodontology 2000. 32(1): 11-23.

Renggli, H. H. and B. Regolati. 1972. Gingival inflammation and plaque accumulation by well-adapted supragingival and subgingival proximal restorations. Helv. Odontol. Acta. 16(2): 99-101.

Rodriguez-Ferrer, H. J., J. D. Strahan and H. N. Newman. 1980. Effect on gingival health of removing overhanging margins of interproximal subgingival amalgam restorations. J. Clin. Periodontol. 7(6): 457-462.

Roman-Torres, C. V. G., S. C. Cortelli, M. W. B. de Araujo, D. R. Aquino and J. R. Cortelli. 2006. A short-term clinical and microbial evaluation of periodontal therapy associated with amalgam overhang removal. J. Periodontol. 77(9): 1591-1597.

Suomi, J. D., J. C. Greene, J. R. Vermillion, J. Doyle, J. J. Change and E. C. Leatherwood. 1971. The effect of controlled oral hygiene procedures on the progression of periodontal disease in adults: Results after third and final year. J. Periodontol. 42(3): 152-160.

Trivedi, S. C. and S. T. Talim. 1973. The response of human gingiva to restorative materials. J. Prosthet. Dent. 29(1): 73-80. 